them from transgressions of which the results may be without remedy. Such good fortune is the portion of but few; for the average human system is rich in self-protective power, and the determination with which it instinctively seeks to maintain its integrity to the last is strikingly shown in two instances. The appearance of unusual health which frequently precedes a seizure, seems to be the final effort which the system makes, under the stimulus of some premonition, to resist the approach of disease. In close analogy with this, is the improvement often noticed in symptoms just before death; a delusive change, which is simply the evidence of one last attempt to rally before yielding in a hopeless struggle.

\title{
A CASE OF POISONING BY FIVE GRAINS OF STRYCHNINE TREATED BY CHLOROFORM INHALATIONS. RECOVERY.
}

By G. W. Copeland, M.D.

As the following case may be of some interest, I will submit it to the profession.

Mr. B., shop-keeper, a middle-aged man of temperate habits, while suffering from depression of spirits, obtained, on Sept. 1st, ten grains of strychnine, representing his intentions were to poison a dog. He secured a room at a hotel, took a dose of laudanum as a preparatory step, and went to bed, intending to swallow the drug as soon as the effects of the opiate were apparent. It appears he fell asleep, and did not awake till half past four in the morning, when he took half the quantity previously mentioned. Some time after, he was seized with convulsions. The occupants of the adjoining rooms, awakened and alarmed by his screams, at length came to his relief. I was called and saw him at 6, A.M. I found him lying in bed; legs and arms extended, his hands firmly clenching the sides of the mattress; intellect clear. He confessed he had taken strychnine. The clothing, by his request, had all been removed, as the slightest touch produced a spasm. I administered twenty grains of sulphate of zinc as soon as it could be obtained. This he swallowed with great difficulty, the contact of the solution with his mouth producing trismus and constriction of the throat. The paroxysms came on every three or four minutes. He was conscious of their approach, and entreated us to hold him, to raise him up, or lift him out of bed, till his body became fixed, his head drawn back and articulation impossible. In this condition of complete opisthotonos, he remained for about a minute, his face livid, and death apparently inevitable. I now resorted to chloroform inhalations, with the happy result of preventing each paroxysm from lasting over a few seconds, or subduing it before the muscles of the back became rigid. So soon as he felt one coming on, I applied the vapor to bis mouth; 
when the muscles were completely relaxed and the breathing natural, I removed it. The convulsions returned regularly till 2, P.M.; the intervals then grew longer till 5 , when the paroxysms entirely subsided. For some time after he regained the use of his hands and arms, the legs could not be touched without producing a shock, as if the poles of a battery had been applied. In the eleven hours, he had used over a pound of chloroform. During the night and next day, I found it necessary to relieve the bladder with the catheter. The following evening - thirty-six hours after I first saw himhe was taken home in his carriage, and a week subsequently he walked to my office, although still suffering from soreness and stiffness of the muscles.

In this case, the sulphate of zinc did not produce emesis, nor did I repeat the dose, feeling confident the drug must already have been absorbed. And here I would state that the treatment given in all the books, viz., "give emetics, and persist in their use till free emesis is produced," should at least be modified. If we do not see the patient till a quarter of an hour after the poison is taken, or if convulsions have set in, emesis will surely do much harm. In a case 1 saw in Philadelphia in 1868, the patient was nauseated with doses of sulphate of zinc and ipecac. Each attempt at emesis produced the most alarming convulsions. With chloroform to ward off the convulsions till the poison is eliminated from the system, deaths from strychnine will be very rare.

50 Marerick Square, Boston.

Oxide of Zinc as a Remedy for the Diarrhea of Infancy and ChildHOOD.-Dr. Brakenridge has carefully tried various methods of treatment in many hundreds of cases of diarrlsœa of infancy and childhood, at the dispensary of the Edinburgh Royal Hospital for sick children. The above remedy he has been led to adopt for the following reasons: First, the disease depends chiefly upon a weak and too impressionable state of the nerve centres presiding over alimentary secretion; secondly, it is correlated to convulsions and other spasmodic diseases; thirdly, it is accompanied by hyperæmia of the secreting surfaces of the alimentary canal. To meet these indications, we must have a remedy which is, firstly, tonic ; secondly, anti-spasmodic; thirdly, astringent. In the oxide of zinc we have these three properties combined. First : as a tonic, it has been said to be to the nervous system what iron is to the blood. Its usefulness in the analogous condition of profuse sweating is well known. Secondly: as an antispasmodic, it is deservedly held in high estimation, and has been found to effect, frequently, a cure in convulsions and other spasmodic diseases. It may, therefore, be depended upon to prevent the occurrence of those nervous diseases which stand correlated to diarrhœa, as the alternative results of such irritations as dentition, worms, \&c., and which may supervene on the rude stoppage of diarrhoea by astringents. Thirdly: its astringent properties are, well known.-Medical Times and Gazette. 